\title{
An Adaptive Interface for Spatial Augmented Reality Workspaces
}

\author{
Wallace S. Lages \\ wlages@vt.edu \\ Center for Human-Computer Interaction \\ Blacksburg, VA
}

\author{
Doug A. Bowman \\ dbowman@vt.edu \\ Center for Human-Computer Interaction \\ Blacksburg, VA
}

\begin{abstract}
A promising feature of wearable augmented reality devices is the ability to easily access information on the go. However, designing AR interfaces that can support user movement and also adjust to different physical environments is a challenging task. We present an interaction system for AR windows that uses adaptation to automatically perform level window movement while allowing high-level user control.
\end{abstract}

\section{CCS CONCEPTS}

- Human-centered computing $\rightarrow$ Mixed / augmented reality; Empirical studies in interaction design; Empirical studies in ubiquitous and mobile computing.

\section{KEYWORDS}

augmented reality, adaptive interfaces, wearable, user interface

\section{ACM Reference Format:}

Wallace S. Lages and Doug A. Bowman. 2019. An Adaptive Interface for Spatial Augmented Reality Workspaces. In Symposium on Spatial User Interaction (SUI '19), October 19-20, 2019, New Orleans, LA, USA. ACM, New York, NY, USA, 2 pages. https://doi.org/10.1145/3357251.3360005

\section{INTRODUCTION}

Everyday AR will likely involve the use of multiple applications or windows, arranged in 3D space by the user. The arrangement can reflect several criteria, such as the priority of each application, the type of content, and the physical layout of the space. Unfortunately, if users walk, manually re-positioning this workspace can be quite inconvenient. If windows are moved as a group, they would need to conform to the new physical layout. In addition, users may also want to access content while walking.

Feiner et al. [4] describe an X11 window system for AR that supports three coordinate frames for windows: 1) display-fixed; 2) surround-fixed; or 3) world-fixed. Both display-fixed and surroundfixed can keep the information in view as the user walks. The surround configuration was emulated by projecting the windows on a virtual sphere that followed the user. Our system uses a similar egocentric reference frame which can also shift dynamically to an allocentric reference frame.

Permission to make digital or hard copies of part or all of this work for personal or classroom use is granted without fee provided that copies are not made or distributed for profit or commercial advantage and that copies bear this notice and the full citation on the first page. Copyrights for third-party components of this work must be honored.

For all other uses, contact the owner/author(s).

SUI '19, October 19-20, 2019, New Orleans, LA, USA

(c) 2019 Copyright held by the owner/author(s).

ACM ISBN 978-1-4503-6975-6/19/10.

https://doi.org/10.1145/3357251.3360005
We present an AR workspace manager for 2D windows positioned in 3D space. Our system enables users to adjust the layout to satisfy different demands and is based on the findings of our prior work on adaptive interfaces [7]. It was designed to allow user control over adaptive behaviors, enabling quick access and fine positioning of information in space.

\section{ADJUSTABLE SPATIAL ADAPTATION}

Our design is centered around two main features: 1) automatically update the information layout as the user moves around and 2) allow users to directly adjust the position of windows in space (either on physical surfaces or around the user).

The adaptive behavior continuously updates the position of the information windows to account for user movement in space and different geometric features of the environment. If the windows are on the walls, the windows will follow the user around without leaving the surface. In that case, the windows will try to maintain the same relative angle from the user and the surface normal (Figure 1 , left). If the windows are floating in space, they automatically align with the velocity vector as the user moves around, so that content is always at the same distance and orientation (Figure 1, right). In both cases, adaptation ignores head direction, allowing users to rotate their heads to see any specific information.

The second feature, position adjustment, works as an override command for the adaptive behaviors. It works in a similar way whether the windows are floating or on physical surfaces. Floating windows use an egocentric reference frame. In adjustment mode, the user can direct the windows to a specific angle by looking to a new direction (Figure 2, left). If the windows are on walls, looking at another point on the wall will move the windows to that spot (Figure 2, right).

Finally, our system also allows users to quickly alternate between surface and floating modes. Our hypothesis was that users could use these features to adjust the workspace layout to better match different conditions. For example, users might cause windows to float around them while walking and then place them on walls when stopped. To make the system more flexible, we also added a "Freeze" function, that disables the adaptation, fixing windows in space.

\subsection{Implementation}

We developed a prototype using Unity 2017 and the Microsoft HoloLens head-mounted display. The HoloLens is a standalone, optical see-through display with an inside-out tracking system. For interaction, we used an xBox 360 Bluetooth controller and native HoloLens gestures. Two different buttons were assigned to direct windows to float or attach to surfaces. Pressing them temporarily 

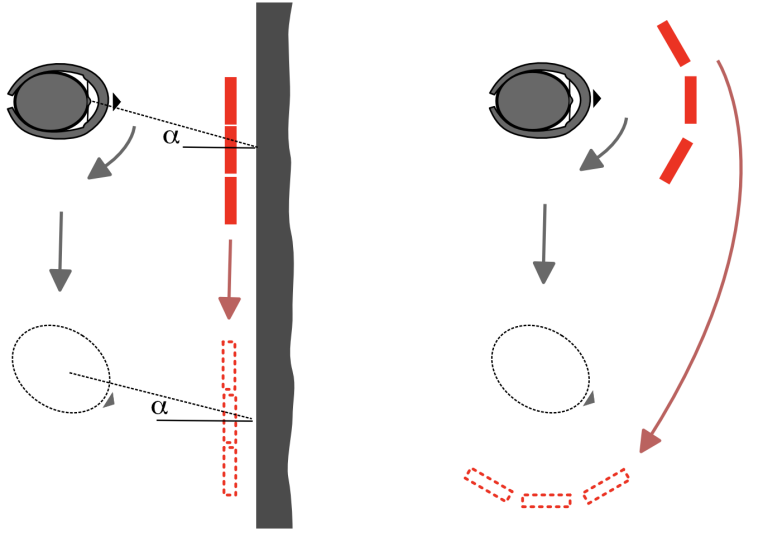

Figure 1: Left: with the windows on the walls, the system tries to maintain the same relative angle between the windows and the user. If the user rotates and translates, the rotation is ignored and the windows just slide on the surface. Right: With floating windows, the windows self-align with the direction of movement. The windows follow the velocity direction and align with the overall trajectory allowing the user to turn the head while walking.
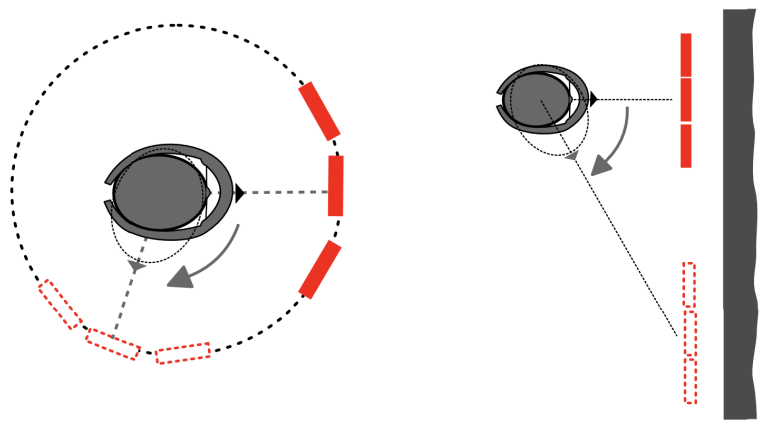

Figure 2: Rotating the head in adjustment mode moves the windows to the corresponding orientation in space (left) or at the intersection with a physical surface (right).

allowed adjusting the position of the windows. We also included a separate button for the "freeze" functionality. Hand gestures were used to drag windows and customize the workspace layout.

Our implementation approach was inspired by behavioral robotics $[2,3]$. Each window follow a gradient descent over a potential field created by surfaces and other physical structures in space $[1,5]$. This approach fast enough for real-time implementation in a real device and works even with incomplete knowledge of the environment. For example, weights can be set so that elements can move towards the user, slide along walls, or to transition between them.
Floating Adaptation State

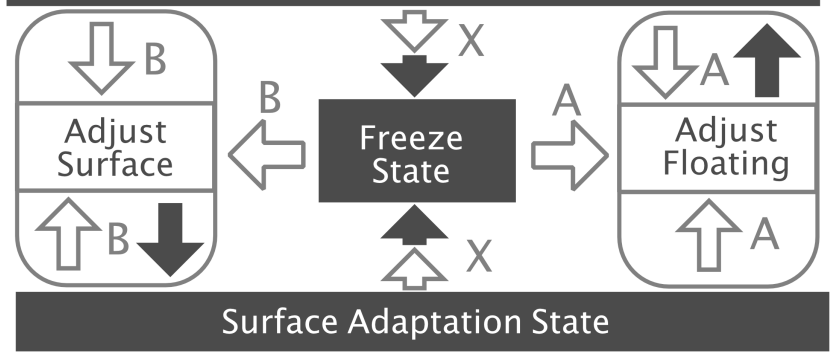

Figure 3: Overview of adjustable behaviors. Arrows indicate a button event (A, B, or $\mathrm{X})$. White arrows indicate button down event and dark arrows, button up. While pressing a button to change state the user can direct the windows to a specific point in space. Pressing and releasing $A$ or $B$ switches windows between floating or surface adaptation.

\subsection{Limitations}

The potential field approach is susceptible to local minima and can create problems in narrow passages [6]. An interface element can also get stuck in an equipotential field created between two walls, or in front of an obstacle. It can also happen if several elements moving together try to reach the same location. This approach works well as proof-of-concept to assess the viability of adaptation, since it allowed us to quickly prototype many different behaviors. In a more sophisticated system, global optimization could be implemented for less flexible and more robust solutions [8].

\section{ACKNOWLEDGMENTS}

The authors gratefully acknowledge funding support from the Immersive Sciences program in the Office of Naval Research and from the Brazilian National Council for Scientific and Technological Development.

\section{REFERENCES}

[1] J Randolph Andrews and Neville Hogan. 1983. Impedance control as a framework for implementing obstacle avoidance in a manipulator. Master's thesis. M. I. T., Dept. of Mechanical Engineering.

[2] Rodney A Brooks. 1991. Intelligence without representation. Artificial intelligence 47, 1-3 (1991), 139-159.

[3] Rodney A Brooks. 1997. From earwigs to humans. Robotics and autonomous systems 20, 2-4 (1997), 291-304.

[4] Steven Feiner, Blair MacIntyre, Marcus Haupt, and Eliot Solomon. 1993. Windows on the World: 2D Windows for 3D Augmented Reality. In Proceedings of the 6th Annual ACM Symposium on User Interface Software and Technology (UIST '93). ACM, New York, NY, USA, 145-155. https://doi.org/10.1145/168642.168657

[5] Oussama Khatib. 1986. Real-time obstacle avoidance for manipulators and mobile robots. In Autonomous robot vehicles. Springer, 396-404.

[6] Y. Koren and J. Borenstein. 1991. Potential field methods and their inherent limitations for mobile robot navigation. In Robotics and Automation, 1991. Proceedings., 1991 IEEE International Conference on. 1398-1404.

[7] Wallace S. Lages and Doug A. Bowman. 2019. Walking with Adaptive Augmented Reality Workspaces: Design and Usage Patterns. In Proceedings of the 24th International Conference on Intelligent User Interfaces (IUI '19). ACM, New York, NY, USA, 356-366. https://doi.org/10.1145/3301275.3302278

[8] Singiresu S Rao and Singiresu S Rao. 2009. Engineering optimization: theory and practice. John Wiley \& Sons. 